\title{
O USO DA TECNOLOGIA NO PROCESSO DIAGNÓSTICO-TERAPÊUTICO: ÓTICA DO ENFERMEIRO E DO USUÁRIO
}

Marisa Ribeiro Bastos Peixoto*

PEIXOTO, M.R.B. O uso da tecnologia no processo diagnóstico-terapêutico: ótica do enfermeiro e do usuário. Rev.Esc.Enf.USP., v.28, n.3, p.257-69, dez. 1994.

São apresentados alguns aspectos relativos ao processo de incorporação tecnológica no setor saúde. Este estudo exploratório teve como objetivo identificar, numa abordagem quantitativa, os pontos positivos e negativos do uso da tecnologia no processo diagnóstico terapêutico, sob a ótica de 35 enfermeiros e 63 usuários dos serviços de saúde. Os resultados mostraram uma tendência em valorizar a utilização dos recursos tecnológicos, tanto sob o ponto de vista dos enfermeiros quanto dos usuários.

UNITERMOS: Tecnologia. Setor Saúde-Brasil.

\section{INTRODUÇÃO}

Grandes transformaçōes tecnológicas assumem fundamental importância na sociedade contemporânea, não só frente às repercussões junto ao processo produtivo, como também por gerar algumas tendências que dão origem a novos formatos organizacionais, novas relações no trabalho, influenciando as qualificações profissionais e relações sociais.

Com o significado de eficiência e qualidade, a tecnologia assume o papel de legitimadora, tanto a nível individual no desempenho de funçōes como também a nível institucional. (PEIXOTO, 1990).

As instituições de saúde, bem como os profissionais que atuam nesse setor, não ficaram alheias a esse processo. Assim como nas organizações produtivas, onde a utilização da tecnologia permite a manutenção de uma posição de igualdade ou mesmo superioridade em relação a concorrentes, como afirma RATTNER (1980), as organizações de prestações de serviços têm o mesmo comportamento, visto que, em sociedades capitalistas, os serviços

\footnotetext{
Enfermeira. Professor Assistente Mestre do Departamento de Enfermagem Básica da Escola de Enfermagem da Universidade Federal de Minas Gerais. Aluna do Programa de Expansảo do Doutorado da Escola de Enfermagem da Universidade de Såo Paulo na Escola de Enfermagem da Universidade Federal de Minas Gerais.
} 
passam, também, a ser considerados bens vendáveis, reproduzindo, assim, a lógica das leis do mercado competitivo.

Num contexto de interesses contrários e posições ideológicas diversas se coloca a questão da tecnologia que, no setor saúde, ou é defendida por seu caráter eficientista e neutro, ou, sob o ponto de vista social, é questionada em relação ao seu acesso e custo elevado, em se tratando de um país caracterizado por profundas desigualdades.

A questão da tecnologia no setor saúde no Brasil assume importância singular, após 1930, em decorrência de pressōes provenientes do processo de industrialização (MAIA, 1984). Segundo o autor, os reflexos da difusão cultural exercida pelos países industrializados passam a ser observados, também, no setor de saúde, tais como, repercussões no ensino médico e na infra-estrutura de saúde.

Nos anos 50, inicia-se a industrialização da medicina, constatando-se uma ampliação da rede hospitalar com ênfase na atenção médica curativa do caráter individual, na especialização e na tecnificação do ato médico. (MAIA, 1984)

$\mathrm{Na}$ década de 60 estabelece-se o discurso hegemônico da racionalidade que, na perspectiva de MAIA (1984), tinha como principal objetivo expandir a assistência curativa no âmbito hospitalar.

A década de 70 foi marcada pelo fortalecimento do setor saúde como um novo setor industrial quando a produção de equipamentos e fármacos passou a observar grandes quantias da renda do país. (MAIA, 1984) No entanto, segundo o autor, essa incorporação tecnológica não significou uma melhoria no nível de saúde da população.

BRAGA; PAULA (1981) apontam a vinculação existente entre os interesses capitalistas no setor saúde e a incorporação tecnológica no processo de produção dos serviços. Segundo os autores, os avanços tecnológicos são fatores "básicos da dinâmica do setor saúde", com repercussões na organização dos serviços hospitalares, ambulatoriais e na prática médica, observando-se, como conseqüência, a expansão do setor industrial de produção de instrumentos e o crescimento de importação de tecnologia para suprir a demanda.

Neste cenário, cabe apontar a atuação do Estado que, através de uma articulação com o setor privado, viabilizou a expansão do capital na assistência à saúde (BRAGA; PAULA, 1981). As políticas de saúde do Estado são, segundo LUZ (1981), vinculadas a um modelo econômico-político e, conseqüentemente no sistema capitalista, às indústrias de medicamentos e equipamentos médicos.

Assim, a utilização da tecnologia no setor saúde está vinculada aos interesses, valores e prioridades do sistema capitalista de produção de bens e serviços e obedece a mesma lógica do capital e da lucratividade. A incorporação tecnológica no processo de produção de serviços de saúde é, assim, uma questão, basicamente, político/econômica que, se por um lado, é sustentada por posições ideológicas de atores sociais que detêm o poder no setor, por outro, é analisada e questionada, face às inúmeras contradições que acarreta no plano social. 
Com o significado nem sempre verdadeiro, de eficiência e qualidade, ideologicamente difundido, a utilização da tecnologia desvirtua as diretrizes e prioridades do setor e ensino dos profissionais de saúde, criando falsas expectativas na sociedade, de resolutividade dos problemas de saúde.

Nossa experiência no ensino e prática da enfermagem, principalmente na área de terapia intensiva, nos levou ao interesse pela investigação sobre o uso da tecnologia no setor saúde, visto que é, cada vez mais, utilizada no processo diagnóstico-terapêutico, passando a influenciar a visão de qualidade da assistência sob o ponto de vista dos prestadores e consumidores dos serviços de saúde (PEIXOTO, 1990).

Este estudo buscou, assim, focalizar essa temática, com o seguinte objetivo:

. Identificar os pontos positivos e negativos do uso da tecnologia no processo diagnóstico-terapêutico, sob o ponto de vista de enfermeiros e de usuários dos serviços de saúde.

\section{METODOLOGIA}

Os dados foram coletados junto a enfermeiros de hospitais privados, gerais, de médio e grande porte da regiāo metropolitana de Belo Horizonte. Para a seleção dos 4 hospitais onde foi desenvolvida a pesquisa foram adotados critérios relativos a número de leitos, grau de densidade tecnológica, serviços assistenciais prestados, categoria e tipo de clientela atendida. Para tal, foram utilizados os critérios de classificação hospitalar do IBGE (1981).

$\mathrm{O}$ universo de enfermeiros foi constituído de 39 profissionais. Dos 39 questionários enviados, foram devolvidos 35 , correspondendo a $89,7 \%$ de retorno.

O estudo da questão proposta pela pesquisa junto aos usuários se caracterizou pela abordagem de uma parcela não probabilística da população de Belo Horizonte.

Foram selecionados 63 respondentes, obedecendo ao critério de indicação por amigos, familiares e de participação voluntária na pesquisa.

Foram elaborados dois questionários, um dirigido aos usuários e outro aos enfermeiros. $O$ instrumento adotado para a coleta de dados junto aos enfermeiros foi constituído de quatro questões, sendo que as três primeiras foram elaboradas com o objetivo de caracterizá-los no que se refere a tempo de formado, área de atuação e qualificação profissional. O questionário destinado aos usuários foi constituído de três questões. As duas primeiras foram elaboradas com o objetivo de caracterizar o perfil dos respondentes em relação à experiência de hospitalização.

A questão aberta de $\mathrm{n}^{2} 4$ do questionário dos enfermeiros e de $\mathrm{n}^{\circ} 3$ do questionário destinado aos usuários foi elaborada com o objetivo de identificar os pontos positivos e negativos da utilização da tecnologia no processo diagnóstico-terapêutico dos pacientes: 
"Citar os principais pontos positivos e negativos da utilização da tecnologia (instrumentos, equipamentos, aparelhos) no processo diagnóstico-terapêutico dos pacientes".

As respostas à questão aberta foram agrupadas em categorias com 0 objetivo de possibilitar uma melhor análise dos resultados.

\section{O USO DA TECNOLOGIA NO PROCESSO DIAGNÓSTICO-TERAPÊU- TICO: A ÓTICA DO ENFERMEIRO}

\section{Perfil dos respondentes}

Dentre os enfermeiros que participaram da pesquisa $97,1 \%$ estão na faixa de até 10 anos de formado e apenas 1 enfermeiro tem de 11 a 20 anos. Dos 35 enfermeiros, $22,9 \%$ têm curso de pós-graduação, referindo-se apenas a cursos de especialização.

No que se refere a área de atuação, constatamos que as quatro áreas mais citadas foram: Enfermagem Médico-Cirúrgica $(20,0 \%)$, Gerência de Enfermagem (20,0\%), Centro de Tratamento Intensivo $(17,1 \%)$ e Enfermagem Pediátrica $(14,3 \%)$.

\section{Pontos positivos e negativos}

Os principais pontos positivos da utilização de tecnologia são, segundo os enfermeiros, a precisão, rapidez e segurança no processo diagnóstico-terapêutico, representando $52,0 \%$ dos pontos positivos citados (TAB.1).

\section{TABELA 1}

Pontos positivos da utilizaçáo da tecnologia no processo diagnóstico-terapêutico, segundo os enfermeiros. Belo Horizonte, 1990.

\begin{tabular}{lrr}
\hline Pontos Positivos & \multicolumn{2}{c}{ Enfermeiros } \\
\hline $\begin{array}{l}\text { Relativos à precisão, rapidez e segurança no processo } \\
\text { diagnostico-terapêutico }\end{array}$ & $N^{0}$ & $\%$ \\
\hline Relativo aos indicadores hospitalares & 53 & 52,0 \\
\hline Relativos ao trabalho do profissional de saúde & 16 & 15,7 \\
\hline Relativos à sobrevida dos pacientes & 15 & 14,6 \\
\hline Relativos a qualidade da assistência & 6 & 5,9 \\
\hline Relativos ao paciente & 6 & 5,9 \\
\hline Outros & 4 & 3,9 \\
\hline TOTAL & 2 & 2,0 \\
\hline
\end{tabular}

A possibilidade de melhorar alguns indicadores hospitalares, tais como: média de permanência, taxa de mortalidade, taxa de infeç̧ão hospitalar e 
custo, é considerada como um dos pontos positivos da utilização da tecnologia no processo diagnóstico-terapêutico, sob o ponto de vista dos enfermeiros.

Significando $14,6 \%$ dos pontos positivos citados, foram indicadas algumas questões relativas ao trabalho do profissional de saúde, ou seja, a possibilidade da tecnologia em melhorar as condições de trabalho, aumentar o nível de informação dos profissionais, constituir-se num incentivo à atualização e possibilitar a elevação do nível dos profissionais, dando-lhes segurança.(TAB.1)

Outros aspectos positivos citados se referiam à capacidade da tecnologia em aumentar a sobrevida dos pacientes $(5,9 \%)$; em melhorar a qualidade da assistência $(5,9 \%)$, bem como em transmitir segurança e tranqüilidade aos pacientes $(3,9 \%)$.

$\mathrm{Na}$ perspectiva dos enfermeiros, os principais pontos negativos da utilização da tecnologia no processo diagnóstico-terapêutico são aqueles relativos à interação profissional/paciente. Segundo os enfermeiros, a tecnologia distancia a relação profissional/paciente, "mecaniza" a assistência, interferindo na visão do "paciente como um todo". (TAB.2)

\section{TABELA 2}

Pontos negativos da utilização da tecnologia no processo diagnóstico-terapêutico, segundo os enfermeiros. Belo Horizonte, 1990.

\begin{tabular}{lrr}
\hline Pontos negativos & \multicolumn{2}{c}{ Enfermeiros } \\
\hline Relativos à interação profissional/paciente & No & $\%$ \\
\hline Relativos ao trabalho do profissional de saúde & 15 & 27,3 \\
\hline Relativos à iatrogenias & 13 & 23,6 \\
\hline Relativos ao custo e acesso & 10 & 18,2 \\
\hline Relativos à qualidade da assistência & 7 & 12,7 \\
\hline Relativos ao uso abusivo & 3 & 5,5 \\
\hline Relativos aos indicadores hospitalares & 3 & 5,5 \\
\hline Relativos ao paciente & 2 & 3,6 \\
\hline TOTAL & 2 & 3,6 \\
\hline
\end{tabular}

Segundo os enfermeiros, a tecnologia interfere no significado e no processo de trabalho do profissional de saúde de forma negativa. A influência da tecnologia no trabalho dos profissionais de saúde é explicada através da ênfase nas especializações e da grande dependência do profissional com relação à tecnologia, ao examinar o paciente, diagnosticar e interpretar os dados coletados. Tal situação, na ótica dos enfermeiros, limita e acomoda 0 profissional que passa, cada vez mais, a condicionar o seu trabalho ao uso da tecnologia.

Foram mencionados pontos negativos relativos às iatrogenias, tais como riscos, falhas e erros no uso da tecnologia, bem como relativos ao alto custo da tecnologia e à dificuldade de acesso à mesma pelas camadas menos privilegiadas da população brasileira. 
Outros aspectos negativos citados, relativos à utilização da tecnologia, se referiam à possibilidade de interferir de forma negativa na qualidade da assistência, de ser usada de forma abusiva, bem como de alterar os indicadores hospitalares, como tempo de permanência e taxa de infecção hospitalar. Foram também citados aspectos negativos relacionados aos pacientes, tais como, medo e insegurança na utilização da tecnologia.

Numa visão global das questões mencionadas pelos enfermeiros, observamos que, das 157 citações apresentadas, $65 \%$ se referiam a pontos positivos da utilização da tecnologia no processo diagnóstico-terapêutico, indicando uma tendência dos enfermeiros em valorizá-la.

\section{O USO DA TECNOLOGIA NO PROCESSO DIAGNÓSTICO-TERAPÊU. TICO: A VISÃO DO USUÁRIO}

\section{Perfil dos respondentes}

Dos 63 respondentes, $33(52,4 \%)$ não tinham experiência de hospitalização, sendo que, daqueles que já haviam sido hospitalizados $(47,6 \%)$, a maioria $(76,8 \%)$ foi submetida a procedimentos cirúrgicos.

\section{Pontos positivos e negativos}

Os principais pontos positivos da utilização da tecnologia no processo diagnóstico-terapêutico são, segundo os usuários, aqueles relativos à precisão, rapidez e segurança, pois $44,7 \%$ dos pontos positivos citados se referiam a estes aspectos (TAB.3)

As questões relativas às potencialidades da tecnologia em manter a vida representaram $19,3 \%$ dos pontos positivos apresentados. Neste grupo foram incluídas respostas que relacionavam a possibilidade da tecnologia em "salvar vidas", "solucionar os problemas de saúde", "controlar os sinais vitais", "curar", "garantir a vida", entre outras da mesma natureza. (TAB.3)

Alguns usuários acreditam que a tecnologia é positiva em si mesma, indica sempre progresso, deve estar sempre presente em termos quantitativos e qualitativos, é sempre "boa", "traz sempre vantagens ilimitadas", são "fundamentais", representando $11,4 \%$ dos pontos positivos apresentados. (TAB.3)

De acordo com os usuários, a tecnologia confere objetividade, rapidez e segurança ao trabalho do profissional de saúde, eliminando ou diminuindo a possibilidade de riscos, erros e falhas do profissional, significando $9,7 \%$ dos pontos positivos mencionados. (TAB.3)

Os pontos positivos da utilização da tecnologia relativos aos pacientes se referiam aos aspectos psicológicos considerados positivos, tais como a 
possibilidade da tecnologia em proporcionar "conforto mental", "segurança", "confiança" e "tranqüilidade" aos pacientes $(8,8 \%)$.

TABELA 3

Pontos positivos da utilização da tecnologia no processo diagnóstico-terapêutico, segundo os usuários. Belo Horizonte, 1990

\begin{tabular}{lrr}
\hline Pontos positivos & \multicolumn{2}{c}{ Usuários } \\
\hline & $\mathrm{N}^{2}$ & $\%$ \\
\hline $\begin{array}{l}\text { Relativos à precisão, rapidez e segurança no } \\
\text { processo diagnóstico-terapêutico }\end{array}$ & 51 & 44,7 \\
\hline Relativos à manutenção da vida & 22 & 19,3 \\
\hline Relativos ao valor da tecnologia & 13 & 11,4 \\
\hline Relativos ao trabalho do profissional de saúde & 11 & 9,7 \\
\hline Relativos ao paciente & 10 & 8,8 \\
\hline Outros & 7 & 6,1 \\
\hline TOTAL & 114 & 100,0
\end{tabular}

Os principais pontos negativos da utilização da tecnologia no processo diagnóstico-terapêutico são, segundo os usuários, aqueles relativos aos riscos operacionais, ou seja, possibilidade de falhas e erros pelo mau uso. As iatrogenias, assim, representaram $35,8 \%$ dos pontos negativos mencionados.(TAB.4)

\section{TABELA 4}

Pontos negativos da utilização da tecnologia no processo diagnostico-terapêutico, segundo os usuários. Belo Horizonte, 1990

\begin{tabular}{lrr}
\hline Pontos negativos & \multicolumn{2}{c}{ Usuários } \\
\hline & $\mathrm{N}^{\circ}$ & $\%$ \\
\hline Relativos as iatrogenias & 24 & 35,8 \\
\hline Relativos aos indicadores hospitalares & 11 & 16,4 \\
\hline Relativos à interação profissional/paciente & 10 & 14,9 \\
\hline Relativos ao custo e acesso & 8 & 11,9 \\
\hline Relativos a imagem da tecnologia & 6 & 9,0 \\
\hline Relativos ao uso abusivo & 5 & 7,5 \\
\hline Outros & 3 & 4,5 \\
\hline TOTAL & 67 & 100,0
\end{tabular}

O risco de contaminação e a maior possibilidade de infecção hospitalar foram citados pelos usuários como pontos negativos da utilização da tecnologia. De acordo com a TAB.4, 16,4\% dos pontos negativos indicados estão incluídos neste grupo.

Na ótica dos usuários, o "distanciamento" e a "frieza" na relação profissional/paciente, bem como a "substituição do profissional pela máquina", a 
transformação do processo diagnóstico-terapêutico em "linha de produção" são pontos negativos da utilização da tecnologia. Constituíram esse agrupamento, 14,9\% dos pontos negativos mencionados pelos usuários.

O alto custo da tecnologia e a dificuldade de acesso da população foram também apontados como pontos negativos do uso da tecnologia, representando 11,95 dos aspectos negativos citados pelos usuários.

De acordo com os usuários, a própria imagem dos aparelhos, equipamentos, (tecnologia) é um fator negativo, pois "é deprimente", "temos medo dela", "é assustadora", entre outros. Fizeram parte desse grupo de respostas, 9,0\% dos pontos negativos citados.

Numa visão global, das 181 respostas apresentadas pelos usuários, constatamos que $63 \%$ se referiam a pontos positivos da utilização da tecnologia no processo diagnóstico-terapêutico.

\section{DISCUSSÃO DOS RESULTADOS E CONCLUSÕES}

Os principais pontos positivos da utilização da tecnologia no processo diagnóstico-terapêutico são, segundo os enfermeiros e usuários, a precisão, rapidez e segurança. Essa potencialidade da tecnologia é também apontada por HOLMES (1990). Segundo o autor, o uso da tecnologia trouxe uma melhora significativa no esclarecimento de diagnósticos. Da mesma forma BRANS (1991) e HILL (1991), descrevendo algumas tecnologias utilizadas na atenção à saúde, apontam a potencialidade da mesma em tornar rápido, preciso e seguro os procedimentos diagnósticos e terapêuticos, melhorando a qualidade da assistência. No entanto, concordamos com McCONNELL; MURPHY (1990), quando afirmam que o uso de tecnologias sofisticadas na atenção à saúde não garante uma assistência de qualidade.

São encontrados na literatura estudos que identificam a relação entre o uso da tecnologia e a redução da taxa de mortalidade (HERRERA et al., 1982), bem como do tempo de permanência nos hospitais (QUIVEY, 1990).

A capacidade da tecnologia em influenciar positivamente o trabalho do profissional de saúde foi apontada por enfermeiros e usuários, embora com maior freqüência entre os últimos. NEVES (1992) afirma que as mudanças tecnológicas têm causado grande impacto sobre o trabalho e a qualificação profissional de uma maneira geral. No contexto da enfermagem, SIMPSOM $(1990,1992)$ afirma que a tecnologia, além de superar os limites físicos relacionados ao tempo, espaço e distância, facilita o acesso ao conhecimento e o registro de informaçōes, aspectos fundamentais para uma prática avançada da enfermagem e para a sua sobrevivência na próxima década.

O principal ponto negativo da utilização da tecnologia no processo diagnóstico-terapêutico é, segundo os enfermeiros, o distanciamento na interação profissional/paciente, aspecto também citado pelos usuários.

Segundo KING (1987), a interface entre profissional/paciente está relacionada ao sucesso na assistência aos pacientes, visto que, além de proporcionar conforto, é uma importante forma de tratamento. De acordo com o autor, 
os avanços tecnológicos, cada vez mais, têm distanciado essa interação, o que por um lado isola o paciente em suas expectativas e ansiedades e, por outro, limita o controle e a influência do profissional na assistência. A interação profissional/paciente, mediada pela tecnologia, torna-se uma questão que merece uma preocupação especial por parte dos profissionais de saúde pois, segundo NINOS (1988), os profissionais às vezes se esquecem de que "um ser humano está do outro lado dos tubos, cabos e drenos; atrás de alarmes; e sob as bandagens, frequentemente limitado"(p.1252).

$\mathrm{Na}$ ótica dos usuários, os principais pontos negativos da utilização da tecnologia no processo diagnóstico-terapêutico são as iatrogenias.

Segundo ILLICH (1975), a incorporaçāo da tecnologia na assistência à saúde trouxe danos não só ao homem como também a nível da sociedade, o que denominou iatrogenia clínica e social. As iatrogenias clínicas são representadas por falhas e erros que muitas vezes levam a conseqüência graves ou mesmo à morte. A fascinação pelos avanços técnicos, as técnicas de medicina terminal e a morte sob controle médico são, segundo o autor, sintomas da iatrogenia social.

A possibilidade da tecnologia em influenciar de forma negativa o trabalho do profissional de saúde foi também mencionada pelos enfermeiros. Os estudos que analisam a influenciam da tecnologia no trabalho focalizam a potencialidade da tecnologia em modificar as qualificações, as relações no trabalho, reforçando a divisão do trabalho e alienando o trabalhador. (ACERO, 1982; RODRIGUES et al, 1985).

No que se refere ao trabalho dos profissionais de saúde, segundo BRANS (1991), existe o risco de que a tecnologia substitua o processo racional e o natural desenvolvimento de uma intuição que surge em decorrência de uma longa prática de observação. No presente estudo, os enfermeiros também apontaram a potencialidade da tecnologia em interferir na "habilidade do profissional em diagnosticar", tornando-se "acomodado", um "mero leitor de dados", "induzindo a uma avaliação clínica superficial".

$\mathrm{O}$ alto custo da tecnologia e a dificuldade de acesso para uma camada significativa da população foram apontados como pontos negativos por enfermeiros e usuários. Essa questão tem sido amplamente estudada, não só nos países pobres, onde a atenção médica se torna cara em decorrência do uso indiscriminado de tecnologia, bem como nos países do Primeiro Mundo (JOHNSSON, 1991; FIESTA, 1992; HAILEY; CROWE, 1991; AMERICAN MEDICAL ASSOCIATION, 1992; LAUPACIS et al., 1992; JOHANSEN; RACOVEANU, 1991).

$\mathrm{Na}$ visão dos usuários, a possibilidade de aumentar a taxa de infeç̧ão hospitalar é um ponto negativo da utilização da tecnologia no processo diagnóstico-terapêutico. Segundo FEEG (1991), a realização de técnicas cirúrgicas complexas - entubações, venopunções, entre outros, aumenta a ocorrência de infecção hospitalar, o que confirma a percepção dos usuários.

O uso abusivo de recursos tecnológicos na assistência à saúde, apontado por enfermeiros e usuários, é um aspecto também enfatizado na literatura que adota a tecnologia como tema de análise. Segundo GONZALEZ (1984), o 
uso abusivo das tecnologias médicas é um dos aspectos mais evidentes de uma assistência inadequada, que acarreta, entre outros, uma distribuição injusta de recursos financeiros, gastos desnecessários e efeitos iatrogênicos. Por outro lado, VILARDELL (1990) refere-se ao abuso tecnológico numa visão ética. Sob esse ângulo, o uso abusivo dos recursos tecnológicos pode ser considerado uma exploraçāo física dos pacientes.

Embora tenham sido apontados alguns pontos negativos da utilização da tecnologia no processo diagnóstico-terapêutico, tanto na ótica dos enfermeiros quanto dos usuários, os resultados obtidos neste estudo mostram uma tendência em valorizá-la. Dentre os 338 aspectos relativos a utilização da tecnologia nos procedimentos diagnósticos e terapêuticos mencionados pelos enfermeiros e usuários, $216(63,9 \%)$ referiam-se a aspectos positivos. Isso também foi evidenciado quando os dados dos enfermeiros e usuários foram analisados em separado.

Nos estudos de FLEMING (1979), SIQUEIRA (1988) e PEIXOTO (1990), foi também identificada a importância conferida aos aspectos tecnológicos de assistência, tanto sob o ponto de vista de médicos e enfermeiros, quanto de usuários dos serviços hospitalares.

A tendência em avaliar o uso da tecnologia de forma positiva pode ser compreendida tendo em vista as prioridades dos sistemas capitalistas. Visto que gera lucro e produtividade, o valor da tecnologia é internalizado nos individuos através do processo de socialização e de educação formal.

Analisando a incorporação tecnológica no setor saúde, BRAGA; PAULA (1981) também apontam a vinculação existente entre esta e os interesses capitalistas. Segundo os autores, a tecnologia é um fator básico na dinâmica do setor, com repercussões na organização dos serviços e na prática dos profissionais de saúde, sendo portanto priorizada.

Assim, o valor da tecnologia é estimulado por um sistema profissional e leigo de "marketing". Segundo LANDMAN (1983), são enfatizadas as pesquisas que apontam os resultados positivos de novas tecnologias, novos meios diagnósticos e de tratamento, com uma propagação prematura desses resultados. Esse processo de legitimação da tecnologia atinge, tanto os profissionais de saúde quanto os consumidores dos serviços.

A visão de que a tecnologia tem a possibilidade de manter ou garantir a vida, mencionada por usuários e enfermeiros, pode ser, assim, explicada, visto que, segundo alguns autores, a conseqüência dessa difusāo a nível da sociedade é uma "fé cega na ciência e na tecnologia". Os indivíduos passam a acreditar que a qualidade do tratamento está intimamente associada à utilização de tecnologia sofisticada e de medicamentos recém-lançados (MELLO, 1983).

Finalmente, é importante ressaltar que nossa posição, embora não contrária à utilização da tecnologia no processo diagnóstico-terapêutico, está de acordo com aqueles que adotam uma postura crítico-reflexiva diante da mesma, visto que, a nosso ver, é necessária uma avaliação de suas limitações, benefícios e uma adequação às necessidades da população como um todo. Assim, concordamos com PIOLA; CASTRO (1985); FINEBERG (1986), BAN- 
TA (1985) e MARQUES (1990), quando apontam a necessidade de supervisionar e controlar o uso da tecnologia na área da saúde, buscando racionalizar a aquisição e a incorporação de novas tecnologias.

PEIXOTO, M.R.B. The use of technology in the health system: point of view of nurses and users.

Rev.Esc.Enf.USP., v.28, n.3, p.257-69, dec. 1994.

Some aspects related to the process of technological implementation in the health system are discussed in the paper. The objective of this exploratory study was to identify in a quantitative approach the positive and negative points related to the introduction of new technology for diagnosis. The point of view of 35 nurses and 63 users of the health system was collected. The results demonstrate that there is a tendency to value the use of new technology in the health system.

UNITERMS: Technology. Health System-Brazil.

\section{REFERÊNCIAS BIBLIOGRÁFICAS}

ACERO, L. O impacto das mudanças tecnológicas nas qualificaçōes de mào-de-obra e no emprego: o caso da indústria têxtil. Rev. Adm. Empr., v. 22, n. 4, p. 28-45, 1982.

AMERICAN MEDICAL ASSOCIATION. Joint report of the council on scientific affairs and the council on medical service: technology assessment in medicine. Arch. Int. Med., v. 152, n. 1, p. 46-50, 1992.

BANTA, D.H. The uses of modern technologies: problems and perspectives fror industrialized and developing countries. In: CONFERENCIA INTERAMERICANA SOBRE AVALIAÇĀO TECNOLÓGICA EM SAÚDE, 1985, Rio de Janeiro. Anais. Rio de Janeiro, 1985, p. 29-46.

BRAgA, J.; PAUla, S.G. de. Saúde e Previdência: estudos de politica social. São Paulo, CEBES/HUCITEC, 1981.

BRANS, Y.W. Biomedical technology: to use or not to use?

Clin. Perinatol., v. 18, n. 3, p. 389-401, 1991.

FEEG, V.D. Iatrogenia: the side effects of being sick. Pediatr. Nurs., v. 17, n. 3, p. 226, 264, 1991.

FIESTA, J. Cost, standards, quality and technology. Nurs. Manage, v. 23, n. 2, p. 16-7, 1992.

FINEBERG, H. Evaluación de la tecnologia médica en paises en vias de desarrolo y en paises industrializados. Salud Publica Mex., v.28, n. 3, p. 328-34, 1986.

FLEMING, G.V. Patient satisfaction as a measure of legitimacy. Chicago, 1979. Tese (Doutorado) - Universidade de Chicago - Departamento de Sociologia. 
FUNDAÇĀO INSTITUTO BRASILEIRO DE GEOGRAFIA E ESTATÍSTICA. Cadastro dos estabelecimentos de saúde. IV: Assistência Médico-Sanitária - 1976. Braślia, Secretaria Planejamento da Presidència da República, 1981.

GONZALEZ, J. Sobreusos de tecnologias medicas: racionalidad de la formulación de licosaminas. Acta Med. Colomb., v. 9, n. 4, p. 139-45, 1984.

HAILEY, D.M.; CROWE, B.L. Health technology assessment: an Australian perspective. Med. Prog. Technol., v. 17, n. 2, p. 103-9, 1991.

HERRERA, M. et al. Influencia del progreso tecnologico en los indices perinatologicos en el area central de Santiago. Rev. Chil. Obstet. Ginecol., v. 47, n. 2, p. 78-94, 1982.

HILL, D.W. 25 years of medical technology. Br. J. Hosp. Med., v. 46, n. 4, p. 242-3, 1991.

HOLMES, B. The influence of technology on holistic nursing. Imprint, v. 37, n. 3, p. 55-64, 1990.

ILLICH, I. Nêmeses da medicina: a expropriação da saúde. Rio de Janeiro, Nova Fronteira, 1975.

JOHANSEN, K.S.; RACOVEANU, N.T. Big ticket health technology: is rational utilization possible? Med. Prog. Technol., v. 17, n. 2, p. 85-91, 1991.

JOHNSSON, J. High-tec health care: how much can we afford? Hospitals, v. 65, n. 16, p. 80 , 1991.

KING, R.C. Technology and the doctor/patient relationship. Postgrad Med. J., v. 63, n. 741 . p. 591-2, 1987.

LANDMANN, J. Medicina não é saúde: as verdadeiras causas da doença e da morte. 2. ed. Rio de Janeiro, Nova Franteira, 1983.

LAUPACIS et al. How attractive does a new technology have to be to warrant adoption and utilization? Tentative guidelines for using clinical and economic evaluations. Can. Med. Assoc. J., v. 146, n. 4, p. 143-81, 1992.

LUZ, M.T. As instituições médica no Brasil: instituição e estratégia de hegemonia. Rio de Janeiro, Graal, 1981.

McCONNELL, E. A.; MURPHY, E.K. Nurse's use of technology: an international concern. Int. Nurs. Rev., v. 37, n. 5, p. 331-4, 1990.

MAIA, P.S. Reflexōes sobre o processo de tecnificação da medicina no Brasil. Rev. Adm. Publ. v. 18, n.4, p. $125-54,1984$.

MARQUEZ, M.B. Ciência, tecnologia e saúde. Rev. Adm., v. 25, n. 1, p. 92-107, 1990.

MELO, C.G. de A medicina. Rio de Janeiro, Achiamé, 1983.

NEVES, M. de A. Mudanças tecnológicas: impactos sobre o trabalho e a qualificação profissional. Cad. Pesq., n. 81, p. 45-52, 1992. 
NINOS, N.P. Humanism and technology. Crit. Care Med., v. 16, n. 12, p. 1252-3, 1988.

PEIXOTO, M.R.B. Tecnologia no setor saúde: critério de avaliação de qualidade dos serviços hospitalares. Belo Horizonte, 1990. Dissertação (mestrado) - Faculdade de Ciências Econômicas, Universidade Federal de Minas Gerais.

PIOLA, S.F.; CASTRO, C. de M. A alta tecnologia na medicina: mais saúde ou mais lucros? In: CONFERENCIA INTERAMERICANA SOBRE A AVALIAÇĀO TECNOLÓGICA EM SAÚ. DE, 1985, Rio de Janeiro, Anais., Rio de Janeiro, 1985. p. 227-32.

QUIVEY, M. Advanced medical technology: finding the answers. Int. Nurs. Rev., v. 37, n. 5, p. $329-44,1990$.

RATTNER, H. Tecnologia e sociedade: uma proposta para os países subdesenvolvidos. São Paulo, Brasiliense, 1980.

RODRIGUES, S.B. et al. Microeletrônica e organizaçāo do trabalho no setor de serviços. Rev. Adm. Empr., v. 25, n. 4, p. 5-19, 1985.

SIMPSON, R.L. How to survice the nest decade. Nurs. Manage., v. 21, n. 12, p. 24-5, 1990.

$22,1992$.

SIQUEIRA, M.M. de. Atendimento hospitalar: o que importa para o paciente? Rev. Adm. Publ., v. 22, n. 4, p. 10-6, 1988.

Vilardell, F. Problemas éticos de la tecnologia médica. Bol. Of. Sanit. Panam., v. 108, n. $5 / 6$, p. $399.405,1990$. 